\title{
Chromium directed regio- and stereocontrol: a new chapter in aromatic natural product synthesis
}

\author{
P.J. Dickens, J.P. Gilday, J.T. Negri and D.A. Widdowson* \\ Department of Chemistry, Imperial College, London SW7 2AY, UK.
}

\begin{abstract}
The sequence of ortho-directing abilities of a series of aryl functionalities in arenetricarbonylchromium( 0$)$ complexes has been shown to be different to that in the uncomplexed arenes. The kinetic resolution of the lithiated complexes with homochiral oxazolidinones has been rationalised via a transition state model and applied to the stereocontrolled synthesis of a series of highly functionalised, homochiral phenethylamines.
\end{abstract}

\section{INTRODUCTION}

The many studies of the chemistry of the aromatic ligands of arenetricarbonylchromuim $(0)$ complexes have established the patterns of reactivity expressed in Figure $1^{1}$. The attachment of a tricarbonylchromium unit to one face of an aromatic ring modifies the chemistry of that ring both electronically and spatially.

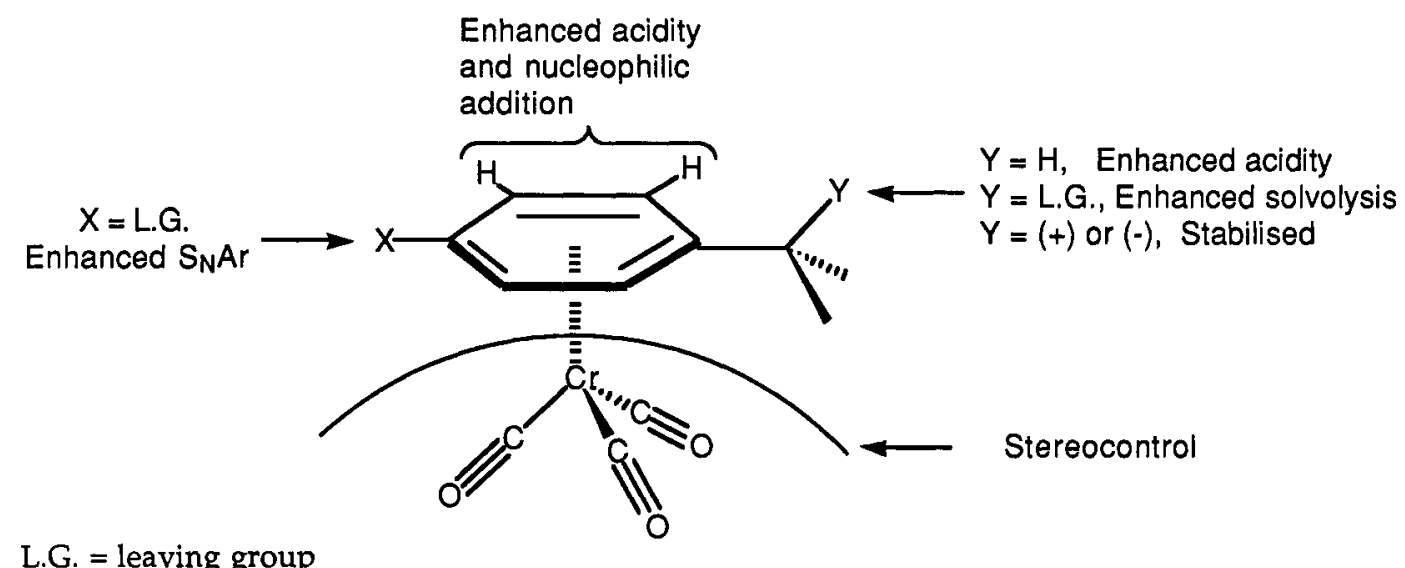

Figure 1

The general effect of the metal carbonyl moiety is that of electron withdrawal, resulting in enhanced acidities of the ring and benzylic protons and an increased propensity to aromatic nucleophilic addition and substitution.

Spatially, the metal occupies one face of the ring and the attack of any reagent on the ring or at a benzylic site is constrained to an approach only from the side anti to the metal. More subtly, such a benzylic attack and the ionisation of a benzylic leaving group has been shown to exhibit a strong stereoelectronic preference and occurs precisely antiperiplanar to the metal atom ${ }^{2}$.

This report will concentrate on two aspects of these attributes, ring deprotonation and stereocontrol, and show how new synthetic strategies can be developed as a consequence of the attachment of the metal. 


\section{REGIOCONTROL: DIRECTED LITHIATION}

The very extensive studies of directed lithiation in aromatic compounds ${ }^{3}$ has resulted in the development of an order of directing abilities for the many functional groups which show this phenomenon (see later).

During our earlier studies an unexpected observation was made that the chromium complex of $p$-fluoroanisole was lithiated at $-78^{\circ} \mathrm{C}$ exclusively ortho to the fluorine atom (Scheme 1) ${ }^{4}$

Scheme 1

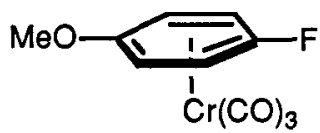

i. $\mathrm{n}-\mathrm{BuLi} / \mathrm{THF} /-78^{\circ} \mathrm{C} / 2 \mathrm{~h}$;

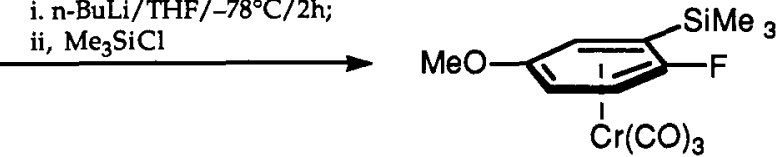

In uncomplexed arenes, fluorine is reportedly a poor ortho directing group 5 and in our hands, under the above conditions, $p$-fluoroanisole gave a $1: 1$ ratio of the monosubstitution products.

It became necessary to determine, therefore, whether this was peculiar to fluorine or whether and how the directing abilities of other groups were modified upon complexation by chromium and we examined complexes containing a range of ortho directing groups.

A series of para disubstituted arene complexes (1) were synthesised and lithiated under standardised conditions (Scheme 2) and reacted with chlorotrimethylsilane which we had established as an efficient quench for the lithio- species ${ }^{4}$.

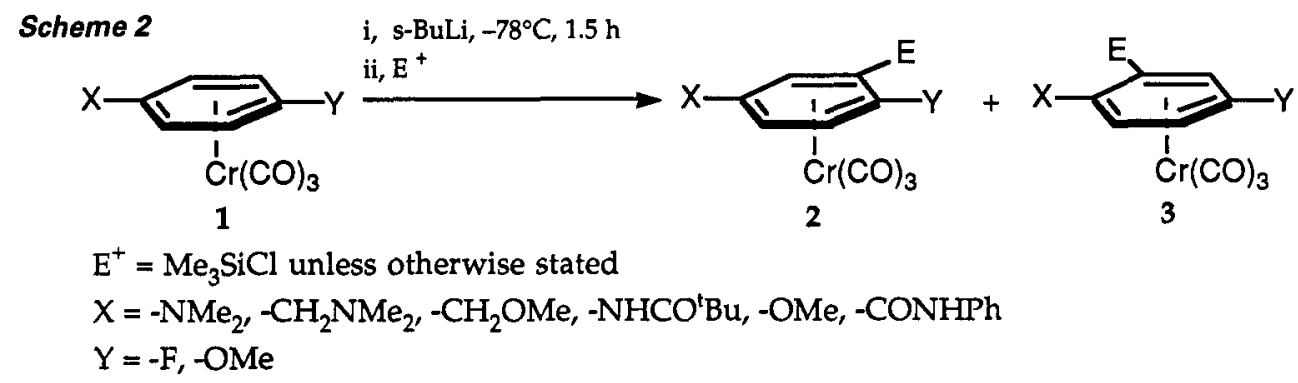

Many of these were run, together with control experiments on the uncomplexed arenes ${ }^{6}$, and some notable examples are given in Figure 2 . Thus $p$-dimethylaminomethylanisole complex $\left(1, \mathrm{X}=\mathrm{CH}_{2} \mathrm{NMe}_{2}, \mathrm{Y}=\mathrm{OMe}\right)$ gave a $1: 1$ mixture of the monosilylated products, yet the uncomplexed arene was substituted exclusively ortho to the aminomethyl group. In contrast, p-t-butoxycarbonylaminoanisole $\left(1, X=\mathrm{NHCO}_{2} \mathrm{Bu}^{t}, \mathrm{Y}=\mathrm{OMe}\right)$ gave a very similar product distribution whether complexed or not.

A clue to the possible reason for the differences comes from the X-ray analysis of the solid
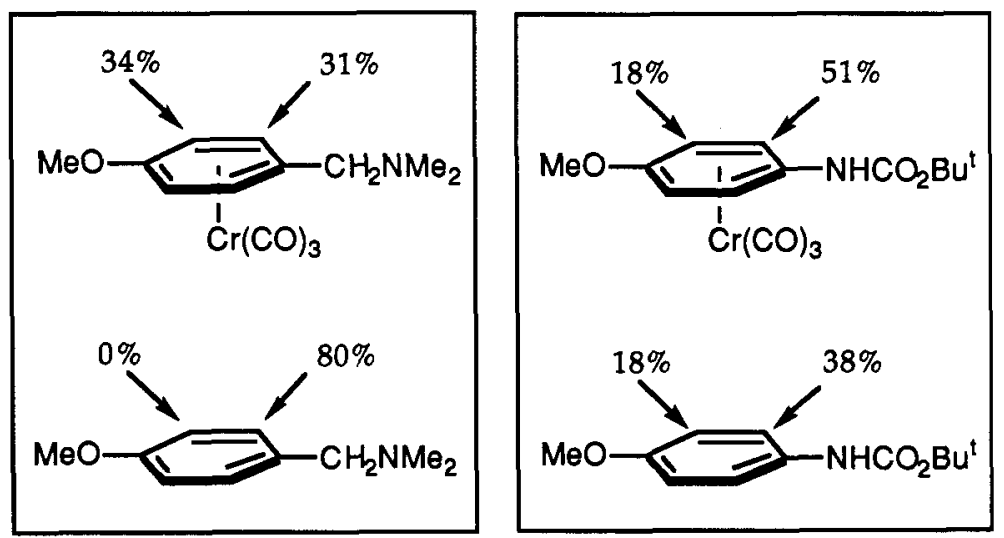

Figure 2 
state structures of the $p$-fluoroanisole complex and related compounds. These show that the methoxy group of $(1, X=F, Y=O M e)$ is almost in the plane of the aryl ring and that whilst the C-F bond length $(1.351 \AA)$ is comparable to that of uncomplexed aryl fluorides $(1.35 \AA) 7$, the aryl $\mathrm{C}-\mathrm{O}$ bond is slightly shortened $\left(1.346 \AA\right.$, cf uncomplexed aryl methyl ethers, $\approx 1.36 \AA^{8}$ ) indicative of increased double bond character in the complex and hence enhanced positive character to the oxygen atom. Furthermore, it is known that aryl amine complexes exhibit a pronounced shortening of the $\mathrm{C}-\mathrm{N}$ bond upon complexation $^{9}(\approx 1.4 \AA$ to $\approx 1.35 \AA)$.

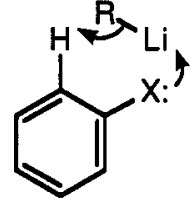

Coordinative Effect<smiles>[X]c1ccccc1[TlH]</smiles>

Inductive Effect

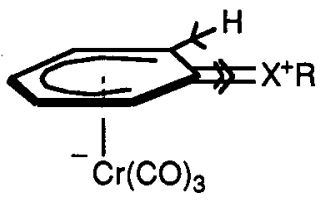

Chromium Effect

Figure 3

Directed ortho lithiations are generally ascribed to a combination of two effects, the coordination of the incoming base and the inductive labilisation of the adjacent ring proton by the electron withdrawing substituent (Figure 3$)^{10}$. These both enhance the kinetic acidities of the ring protons. Fluorine in aryl fluorides is non-coordinating towards incoming bases 10 and the strong back donation from the fluorine non-bonding electrons to the aryl ring, cited as the reason for the short $\mathrm{C}-\mathrm{F}$ bond length ${ }^{11}$, would tend to counteract the inductive effect of the fluorine atom by enhancing the electron density on the ring.

As judged by the $\mathrm{X}$-ray data, the $\mathrm{C}-\mathrm{F}$ bond length is unchanged on complexation to the metal. In the sequence from fluorine to nitrogen, the increasing bond shortening upon complexation indicates an increasing back donation and a progressive reduction in the coordinating ability of the heteroatoms.

In contrast, the effect in the $\sigma$ framework will be to increase the the inductive effect more especially as the negative charge is not located on the ring but remote from it on the metal carbonyl unit. Thus there appears to be a particular 'chromium effect' (Figure 3), a twofold modification of the reactivity which differs from the consequence of adding a competitive ligand such as $N, N, N^{\prime} N^{\prime}$-tetramethylethylenediamine (TMEDA) ${ }^{12}$. This explains why the methoxy group in $p$-dimethylaminomethylanisole complex shows comparable activation to the aminomethyl group, even though the latter is a more powerful director in the parent arene in the presence of TMEDA 12 .

The secondary amide group is one of the most powerful ortho directors in aromatic systems ${ }^{13}$. The aromatic ring of the $o$-fluorobenzanilide complex proved to be so electron deficient that kinetic lithiation with alkyl lithiums was not possible. Addition of the alkyl anion to the ring was the only process observable. However, lithiation under thermodynamic conditions with lithium diisopropylamide was possible. Quenching of the anion with chlorotrimethylsilane gave silylation next to fluorine as the only detectable substitution product (Scheme 3).

Scheme 3
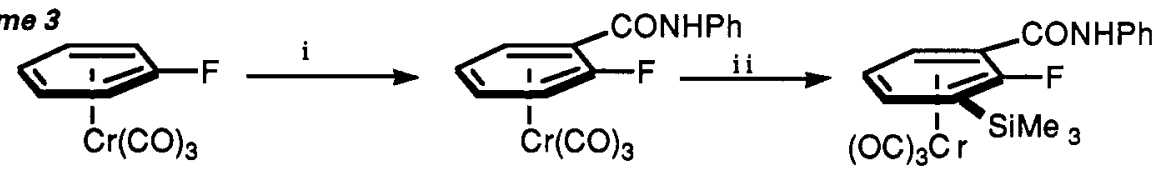

$57 \%$

i; $\mathrm{n}-\mathrm{BuLi} / \mathrm{THF} /-78^{\circ} \mathrm{C}, \mathrm{PhNCO}$.

ii; $\mathrm{LDA}(2 \mathrm{eq}.) / \mathrm{THF} /-78^{\circ} \mathrm{C}, \mathrm{Me}_{3} \mathrm{SiCl}$. 
Based upon these results, we can now construct a new order of directing abilities for the complexes:compared with:-

$$
-\mathrm{F}>(-\mathrm{CONHR})>-\mathrm{NHCOR}>-\mathrm{CH}_{2} \mathrm{NR}_{2} \approx-\mathrm{OMe}>>-\mathrm{CH}_{2} \mathrm{OMe}>-\mathrm{NR}_{2},-\mathrm{SR}
$$

for the uncomplexed arenes.

$$
-\mathrm{CONR}_{2}>-\mathrm{SO}_{2} \mathrm{NR}_{2}>-\mathrm{NHCOR}>-\mathrm{CH}_{2} \mathrm{NR}_{2}>-\mathrm{OMe}>-\mathrm{NMe}_{2} \approx-\mathrm{F}
$$

The use and power of this new order has been illustrated by the synthesis of polyfunctionalised aromatics from $o-, m$ - and $p$-fluoroanisole complexes ${ }^{4}$ and provided new strategies for the synthesis of highly functionalised aromatic natural products and related compounds as exemplified by the phytoalexins of Morus spp. ${ }^{14}$ and the functionalised $\beta$ phenethylamines as set out below.

\section{STEREOCONTROL: KINETIC RESOLUTION}

1,2-Diheterofunctionalised benzenetricarbonylchromium( 0 ) complexes are asymmetric but not readily obtained in homochiral form 15 . We sought to assess their potential for diastereoselection in their reaction with chiral reagents (Scheme 4).

Seebach's chiral 5 -oxazolidinones 16 were the chosen reagents because of the diversity of their potential for natural product and bioactive molecule synthesis. In the event, successful reaction required the use of an equivalent of a Lewis acid and of the many studied, boron trifluoride etherate proved to be the most effective. Without this, the lithiated complex simply deprotonated the substrate. It is presumed that the much less basic boron ate complex is the reacting species. In addition, it was found necessary to use a more bulky acyl group on $\mathrm{N}-3$ in order to avoid attack at the amide carbonyl group.

Scheme 4
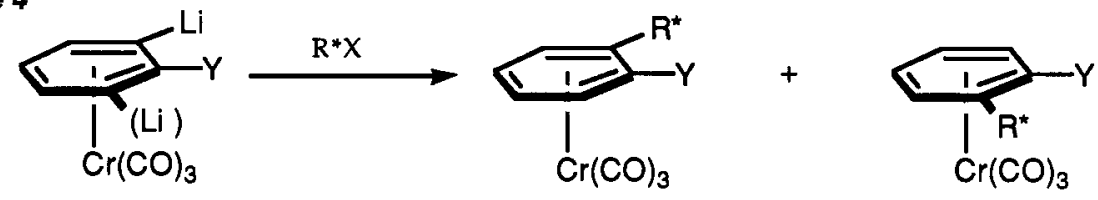

$R,(S)$

$R^{*} X=$ chiral reagent

The reaction of 2-lithiofluorobenzene complex with the alanine derived synoxazolidinone (Scheme 5) gave the adducts in $69-80 \%$ yield without diastereoselection. The structures of these complexes were determined by a single crystal $\mathrm{X}$-ray analysis of the $(S, S)$ isomer.

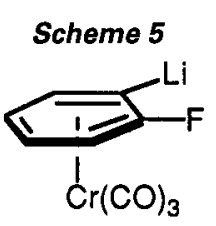

$(\mathrm{R}, \mathrm{S})$

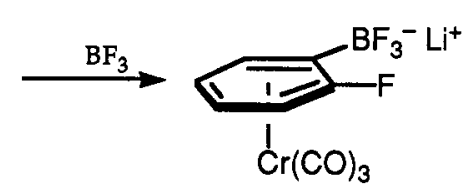

$(\mathrm{R}, \mathrm{S})$

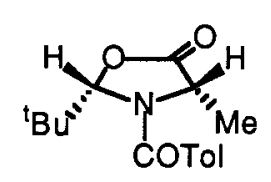

(4-S)

0.5 equiv.

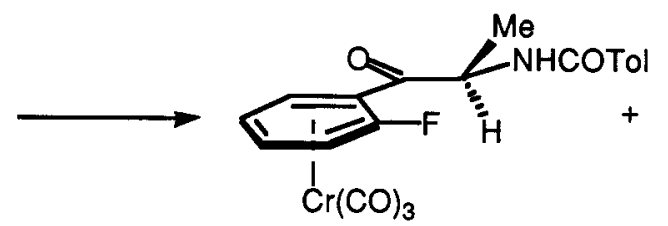

$(S, S)$

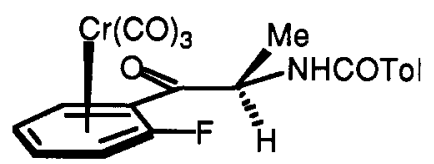

$(R, S)$

1

With more bulky ortho directing groups, the diastereoselection increased markedly. Thus 2-lithioanisole complex gave a $3: 1$ ratio of diastereomers and the 2-lithiopivalamidobenzene complex a 10.4: 1 (Scheme 6), the structures of which were determined by chemical correlation with the single crystal $X$-ray analysis of the analogous 2-fluoro- complex above. 


\section{Scheme 6}<smiles>CC(C)(C)NC1=CC2C=CC(C(C)(C)C)(C1)C2</smiles>

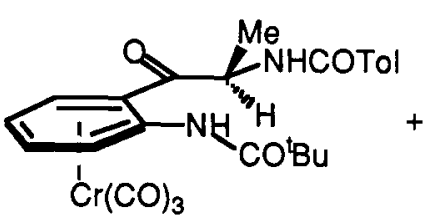

$(S, S)$

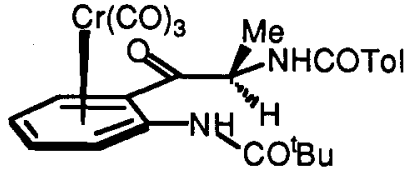

$(R, S)$

10.4

Although stereodifferentiation by a large group adjacent to lithium can be readily appreciated, more surprising was the observation of stereodifferentiation by a remote large group (Scheme 7).

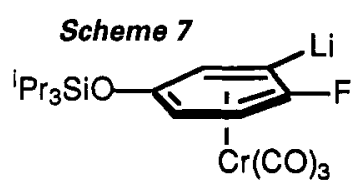

$(R, S)$

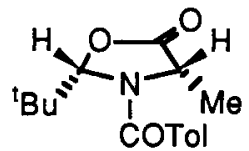

0.5 equiv.

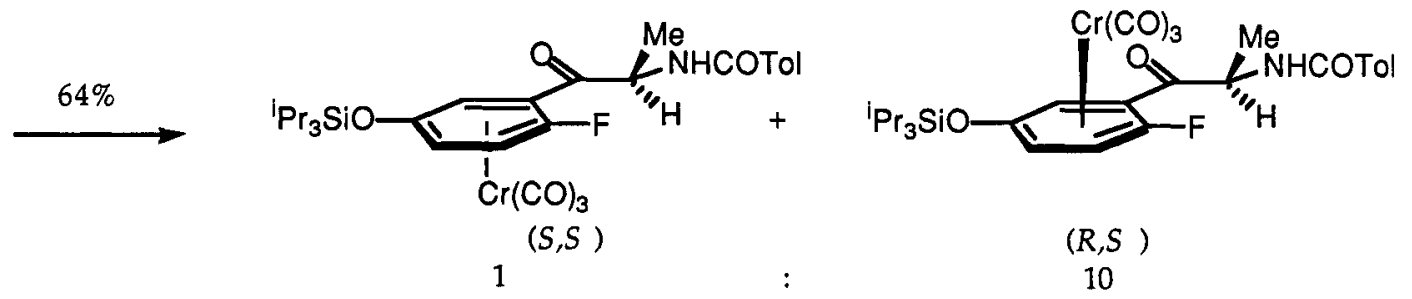

All of these results can be rationalised in terms of the transition states given in Figures 4 and 5.

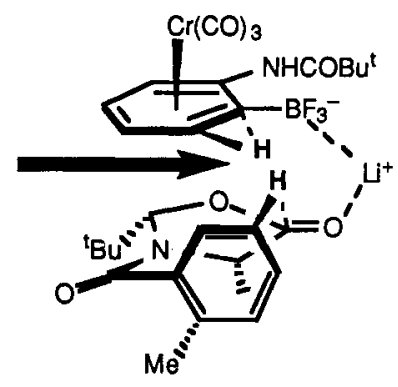

$(S, S)$

Favoured

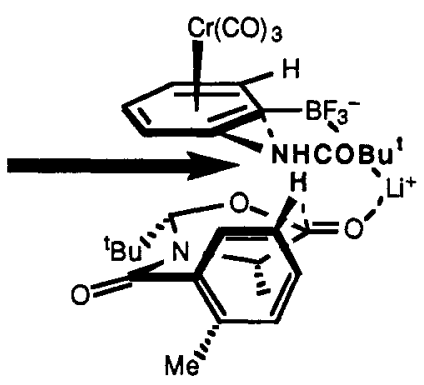

$(R, S)$

Disfavoured

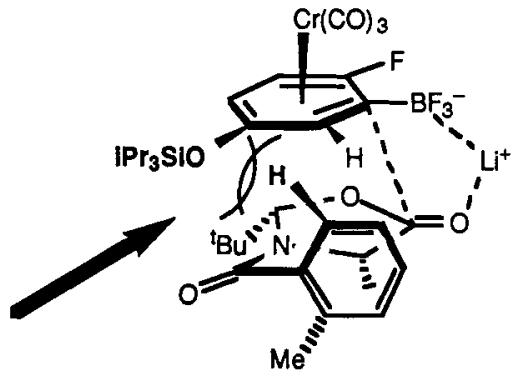

$(S, S)$

Figure 4

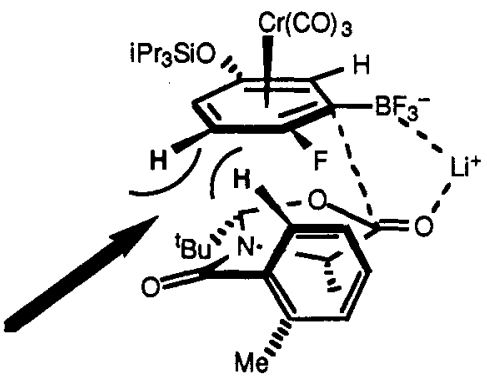

$(R, S)$

Disfavoured Figure 5

Favoured 
Access to these homochiral complexes allowed application of benzylic stereocontrolled reactions of chromium tricarbonyl complexes to a chiral synthesis of ephedrines (Scheme 8).

Thus the $(R, S)$ - isomer gave a single diastereomer with the erythro- configuration.

\section{Scheme 8}

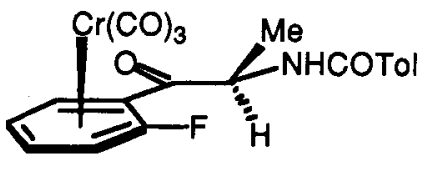

$(R, S)$

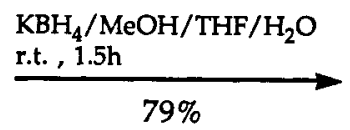

$79 \%$

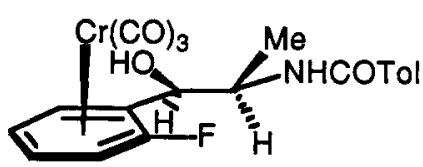

$(R, S, S)$

Ephedrines

The fact that the chromium unit is known to control completely the stereochemistry of reactions at the benzylic site ${ }^{2}$, taken together with our observation that larger side chains (from methionine or valine) on the oxazolidinone served to diminish the proportion of the threo ( $\psi$-ephedrine) series from the reduction of the $(S, S)$ - isomer, shows that the reaction occurs via an equilibrating pair of conformers (Scheme 9 ).

\section{Scheme 9}

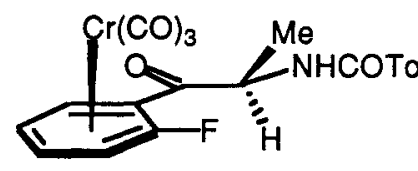

$(R, S)$

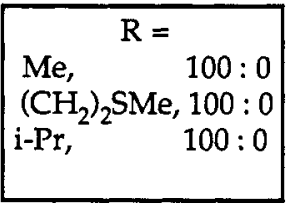

erythro-

(Ephedrines)
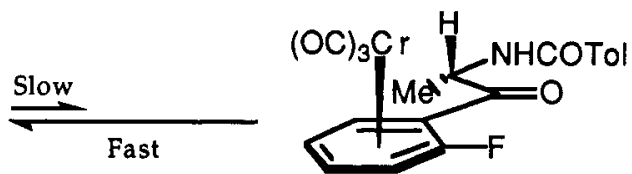

\section{Matched Stereocontrol}

These results can be summarised as shown in Figure 6 .

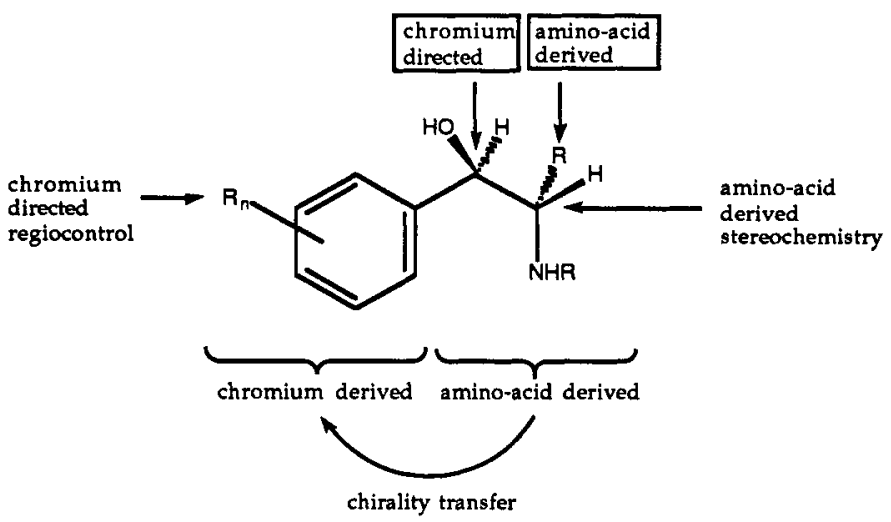

Figure 6

Thus we have reached the first stage of a programme of natural product and related compound synthesis. By a combination of the two features described here, the potential exists to synthesise a wide range of highly functionalised homochiral phenethylamines. The chromium complexes are also being applied to the synthesis of more phytoalexins, and a range of indolic alkaloids and antibiotics ${ }^{17}$. 


\section{REFERENCES}

1. For recent examples see: a. E.P. Kundig, V. Desobry, D.P. Simmons and E. Wenger, J. Am. Chem. Soc., 111, 1804 (1989); b. L. Keller, K. Times-Marshall, S. Behar and K. Richards, Tetrahedron Lett., 26, 3373 (1989); c. R.D. Reike, K.P. Daruwala and M. W. Forkner, J. Org. Chem., 54, 21 (1989); d. S.G. Davies and C.L. Goodfellow, J. Chem. Soc. Perkin Trans. 1, 192 (1989); e. M. Uemura, T. Minami, K. Hirotsu and Y. Hayashi, J. Org. Chem., 54, 469 (1989) and references there cited.

2. S.J. Coote and S.G. Davies, J. Chem. Soc., Chem. Commun., 648 (1988); S. Top and G. Jaouen, J. Org. Chem., 46, 78 (1981).

3. H.W. Gschwend and H.R. Rodriguez, Org. React., 26, 1 (1979); B.J. Wakefield 'The Chemistry of Organolithium Compounds' Pergamon Press, Oxford, 1974. For recent studies of directed o-lithiations see:- M. Iwao, T. Lihama, K.K. Mahalanabis, H. Perrier and V. Snieckus, J. Org. Chem. 54, 26 (1989); G.D. Figuly, C.K. Loop, and J.C. Martin J. Am. Chem. Soc., 111, 654 (1989); E. Block, V. Eswarakrishnan, M. Gernon, G. Ofori-Okai, C. Saha, K. Tang and J. Zubieta, J. Am. Chem. Soc., 111, 658 (1989).

4. J.P. Gilday and D.A. Widdowson, J. Chem. Soc., Chem. Commun., 1235 (1986); ibid., Tetrahedron Lett., 27, 5525 (1986).

5. D.C. Furlano, S.N. Calderon, G. Chen and K.L. Kirk, J. Org. Chem., 53, 3145 (1987).

6. J.P. Gilday, J.T. Negri and D.A. Widdowson, Tetrahedron, 45, 4605 (1989).

7. cf. L. Nygaard, I. Bojesen, T. Pedersen and J. Rastrup-Andersen, J. Mol. Struct., 2, 209 (1968).

8. M. Colapietro, A. Domenicano and G.P. Ceccarini, Acta Cryst., 35B, 890 (1979).

9. The bond lengths used in this discussion, other than our own data, are taken from structures held at the Cambridge Crystallographic Database; $c f$. A. Domenicano and A. Vaciago, Acta Cryst., B35, 1392 (1979).

10. W. Bauer and P.von R. Schleyer, J. Am. Chem. Soc., 111, 7191 (1989).

11. For a full discussion see: W.A. Sheppard and C.M. Sharts, 'Organic Fluorine Chemistry', W.A. Benjamin, New York, 1969, pp 18-49.

12. D.W. Slocum and C.A. Jennings, J. Org. Chem., 41, 3653 (1976).

13. P. Beak, A. Tse, J. Hawkins, C. Chen and S. Mills, Tetrahedron, 39, 1983 (1983).

14. J.M. Clough, I.S. Mann and D.A. Widdowson, Tetrahedron Lett., 28, 2645 (1987)

15. But see: S.G. Davies and C.L. Goodfellow, SynLett.,(1), 59 (1989); refs. 1d and 1e and references cited there.

16. D. seebach, M. Boes, R. Naef and B.W. Schweizer, J. Am. Chem. Soc., 105, 5390 (1985).

17. I.S. Mann, PhD Thesis, London University, 1988; M.J. Dickens, PhD Thesis, London University, 1989. 